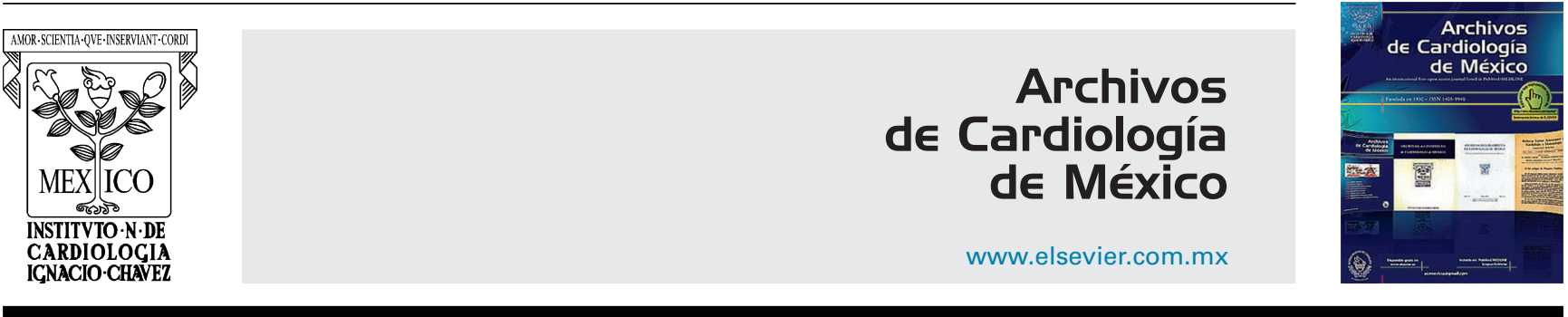

INVESTIGACIÓN CLÍNICA

\title{
Análisis de la cultura de seguridad en una Unidad de Cardiología gestionada por procesos
}

\author{
Rafael Raso-Raso ${ }^{a, *}$, Joaquín Uris-Selles ${ }^{\mathrm{b}}$, Andreu Nolasco-Bonmatí ${ }^{\mathrm{b}}$, \\ Guillermo Grau-Jornet ${ }^{a}$, Rosa Revert-Gandia ${ }^{a}$, Rebeca Jiménez-Carreño ${ }^{a}$, \\ Ruth M. Sánchez-Soriano ${ }^{a}$, Carlos I. Chamorro-Fernández ${ }^{a}$, Elvira Marco-Francés ${ }^{a}$ \\ y José V. Albero-Martínez ${ }^{\text {a }}$
}

\author{
a Unidad de Cardiología, Hospital Virgen de los Lirios, Alcoy, Alicante, España \\ b Departamento de Enfermería Comunitaria, Medicina Preventiva y Salud Pública e Historia de la Ciencia, \\ Facultad de Ciencias de la Salud, Universidad de Alicante, San Vicente del Raspeig, España
}

Recibido el 1 de noviembre de 2016; aceptado el 7 de marzo de 2017

\author{
PALABRAS CLAVE \\ Cultura de seguridad; \\ Gestión de la calidad; \\ Seguridad del \\ paciente; \\ Hospital; \\ Cardiología; \\ España
}

\begin{abstract}
Resumen La cultura sobre seguridad se perfila como uno de los requisitos para evitar la aparición de efectos adversos, sin embargo, no se ha estudiado en el ámbito de la cardiología. El objetivo es evaluar la cultura de seguridad en una unidad de cardiología que tiene implantado y certificado un sistema integrado de gestión de calidad y riesgos para la seguridad del paciente. Método: Se realizó un estudio observacional trasversal en 2 años consecutivos utilizando la encuesta Hospital Survey on Patient Safety Culture de la «Agency for Healthcare Research and Quality» en su versión española (42 ítems agrupados en 12 dimensiones) sobre todo el personal. Se comparó el porcentaje de respuestas positivas de cada dimensión en 2014 y 2015, así como con los datos a nivel nacional y en EE. UU., siguiendo las recomendaciones establecidas.

Resultados: La valoración global, sobre un máximo de 5, fue de 4.5 en 2014 y de 4.7 en 2015. Identificamos 7 dimensiones como fortaleza. Las peor valoradas fueron: Dotación de personal, Apoyo de la gerencia y Trabajo en equipo entre unidades. La comparación mostró superioridad en todas las dimensiones a nivel nacional, y en 8 respecto a los datos del registro americano. Conclusiones: La cultura de seguridad en una unidad de cardiología con un sistema integrado de gestión de calidad y riesgos y seguridad del paciente es elevada, superior a la nacional en todas sus dimensiones y en la mayoría de ellas respecto al registro de EE. UU.

(C) 2017 Instituto Nacional de Cardiología Ignacio Chávez. Publicado por Masson Doyma México S.A. Este es un artículo Open Access bajo la licencia CC BY-NC-ND (http://creativecommons. org/licenses/by-nc-nd/4.0/).
\end{abstract}

\footnotetext{
* Autor para correspondencia. C/Oliver 37. Escalera A Puerta 7C. 03802 Alcoy (Alicante). España. Correo electrónico: Rrasoraso@gmail.com (R. Raso-Raso).
} 


\section{KEYWORDS}

Safety culture;

Quality management;

Patient safety;

Hospital;

Cardiology;

Spain

\section{Analysis of the safety culture in a Cardiology Unit managed by processes}

Abstract Safety culture is one of the requirements for preventing the occurrence of adverse effects. However, this has not been studied in the field of cardiology. The aim of this study is to evaluate the safety culture in a cardiology unit that has implemented and certified an integrated quality and risk management system for patient safety.

Methods: A cross-sectional observational study was conducted in 2 consecutive years, with all staff completing the Spanish version of the questionnaire, "Hospital Survey on Patient Safety Culture" of the "Agency for Healthcare Research and Quality", with 42 items grouped into 12 dimensions. The percentage of positive responses in each dimension in 2014 and 2015 were compared, as well as national data and United States data, following the established rules.

Results: The overall assessment out of a possible 5, was 4.5 in 2014 and 4.7 in 2015. Seven dimensions were identified as strengths. The worst rated were: staffing, management support and teamwork between units. The comparison showed superiority in all dimensions compared to national data, and in 8 of them compared to American data.

Conclusions: The safety culture in a Cardiology Unit with an integrated quality and risk management patient safety system is high, and higher than nationally in all its dimensions and in most of them compared to the United States.

( 2017 Instituto Nacional de Cardiología Ignacio Chávez. Published by Masson Doyma México S.A. This is an open access article under the CC BY-NC-ND license (http://creativecommons. org/licenses/by-nc-nd/4.0/).

\section{Introducción}

Las organizaciones con una alta cultura de seguridad se caracterizan por una comunicación basada en la confianza mutua, comparten la percepción de la importancia de la seguridad y confían en la eficacia de las acciones preventivas ${ }^{1}$.

La cultura sobre seguridad del paciente en las instituciones sanitarias se perfila como uno de los requisitos esenciales para evitar en lo posible la aparición de efectos adversos, y poder aprender de los errores, para rediseñar los procesos de manera que no se vuelvan a producir. En este sentido la consecución de una adecuada cultura de seguridad es señalada como la primera de las «buenas prácticas» o recomendaciones para mejorar la seguridad del paciente en un informe publicado en 2003 por el National Quality Forum de EE. UU. ${ }^{2}$, hecho que fue reforzado en su actualización de $2007^{3}$. En él se indica que, además de la medición de la cultura de seguridad, el feed-back de los resultados, y las intervenciones consecuentes son elementos imprescindibles para su implementación. Esta es también una de las conclusiones de la Declaración del Consejo de Europa en Varsovia, que recomienda a los países miembros estrategias de abordaje del problema de la seguridad de los pacientes a escala nacional ${ }^{4}$. En España un taller de expertos promovido por la Agencia de Calidad del Sistema Nacional de Salud en el año 2005 para definir la estrategia de seguridad del paciente, en su documento de conclusiones, destaca la necesidad de alcanzar una sensibilización cultural de los profesionales en prevención de riesgos (cultura de seguridad) ${ }^{5}$. El Plan de Calidad del Sistema Nacional de Salud, en su última edición de 2010, establece que uno de los objetivos de la estrategia para mejorar la seguridad de los pacientes es «promover y desarrollar una cultura de seguridad del paciente entre los profesionales y los pacientes, en cualquier nivel de la asistencia sanitaria» ${ }^{6}$.

Dos revisiones realizadas ${ }^{7,8}$ para conocer las herramientas disponibles de evaluación de la cultura de seguridad en una organización coinciden en 2 cuestionarios como los más útiles: el Safety Attitudes Questionary y el Hospital Survey on Patient Safety Culture (HSOPSC) ${ }^{10}$. En 2004 la Agency for Healthcare Research and Quality (AHRQ) de EE. UU. desarrolla el HSOPSC, ampliamente utilizado en cientos de hospitales; dicho cuestionario ha sido adaptado en distintos países y la propia AHRQ dispone de una versión para los hispanoparlantes. En España, la Agencia de Calidad del Sistema Nacional de Salud publica en el año 2005 la versión española del HSOPSC ${ }^{11}$ como fruto de una anterior cooperación entre el Ministerio de Sanidad y Consumo y la Universidad de Murcia. Se trata de una herramienta autoadministrada válida y fiable, que adapta al contexto español la encuesta original de la AHRQ de EE. UU., y que puede ser utilizada para medir este aspecto de la cultura de las organizaciones, identificar los aspectos en que habría que incidir para mejorarla y poder monitorizar su evolución.

En EE. UU., con mayor tradición y desarrollo del tema, la $A H R Q$ publica periódicamente (cada 2 años), un registro con los resultados obtenidos en distintos hospitales con el fin de poder establecer comparaciones y evaluar la situación concreta de cada hospital. El último registro publicado (2014) muestra los datos de 653 hospitales de EE. UU. ${ }^{12}$. Recientemente se han comparado los resultados de 5 servicios de cirugía cardiaca de EE. UU. con los de la base de datos, obteniendo así fortalezas y oportunidades de mejora ${ }^{13}$. En otro estudio realizado en Michigan, se demuestra que a mayor cultura de seguridad menor tasa de complicaciones en cirugía bariátrica ${ }^{14}$. Esta herramienta, utilizada con relativa frecuencia en EE. UU. ${ }^{15,16}$, también ha sido empleada en 
distintos hospitales europeos (belgas ${ }^{17}$, noruegos ${ }^{18}, \operatorname{turcos}^{19}$ y en algunas unidades de cuidados intensivos de hospitales del Reino Unido ${ }^{9}$ ).

En España se utiliza esta herramienta en 2008 para analizar la cultura de seguridad a nivel nacional ${ }^{20}$, publicando un informe sobre cultura de seguridad del Ministerio de Sanidad e lgualdad Social en el año $2009^{21}$. En el año 2013 se publica un estudio en el que se analiza 173 encuestas HSOPSC, en un área de salud andaluza ${ }^{22}$. Por último, en 2015 se publica el primer estudio del país que analiza la cultura de seguridad en atención primaria ${ }^{23}$. Sin embargo nunca se ha estudiado la cultura de seguridad en el ámbito de la cardiología.

Existe un interés creciente por parte de las especialidades de medicina interna en prestar una asistencia de calidad a nuestros pacientes ${ }^{24}$. Por otra parte ya existe evidencia contrastada de que la gestión por procesos puede mejorar el funcionamiento de las unidades en sanidad, con el consiguiente beneficio para el paciente y los trabajadores ${ }^{25-27}$. La norma UNE 179003:2013 sobre gestión de riesgos para la seguridad del paciente agrupa una serie de procesos para la organización, aplicación y evaluación en este sentido, en un intento de asegurar una asistencia sin riesgos evitables a los pacientes. Dicha norma esta poco implantada en España y en los países latinoamericanos.

El objetivo del presente trabajo fue evaluar la cultura de seguridad en una Unidad de Cardiología que tiene definido, implantado y certificado un sistema integrado de gestión de calidad y riesgos para la seguridad del paciente.

\section{Material y métodos}

\section{Población a estudio}

La Unidad de Cardiología está ubicada en un hospital general de 305 camas que atiende a una población aproximada de 150,000 habitantes. En el momento del estudio contaba con un total de 32 trabajadores teniendo en cuenta el personal facultativo, enfermería, auxiliares y personal de apoyo. La unidad certificó su sistema de Gestión de la Calidad en 2010 de acuerdo a la norma ISO 9001-2008. Posteriormente en abril de 2014 integró el sistema de gestión de riesgos y seguridad del paciente certificándose también con la norma UNE 179003.

\section{Cuestionario del estudio}

Se realizó un estudio observacional trasversal en 2 años consecutivos. Para ello se utilizó la encuesta HSOPSC de la AHRQ en su versión española ${ }^{11}$. El cuestionario cuenta con un total de 42 ítems distribuidos en 12 dimensiones: Frecuencia de eventos notificados (agrupa 3 ítems), Percepción de seguridad (agrupa 4 ítems), Expectativas y acciones de la dirección/supervisión de la unidad/servicio que favorecen la seguridad (agrupa 4 ítems), Aprendizaje organizacional/mejora continua (agrupa 3 ítems), Trabajo en equipo en la unidad/servicio (agrupa 4 ítems), Franqueza en la comunicación (agrupa 3 ítems), Feed-back y comunicación sobre errores (agrupa 3 ítems), Respuesta no punitiva a los errores (agrupa 3 ítems), Dotación de personal (agrupa 4 ítems), Apoyo de la gerencia del hospital en la seguridad del paciente (agrupa 3 ítems), Trabajo en equipo entre unidades (agrupa 4 ítems) y Problemas en cambios de turno y transiciones entre servicios/unidades (agrupa 4 ítems). La respuesta a cada ítem tiene 5 opciones de 1 a 5 , donde 1 corresponde a «muy en desacuerdo/nunca» y 5 corresponde a «muy de acuerdo/siempre». También se analizó una serie de variables sociolaborales: antigüedad en la unidad, antigüedad en la profesión y número de horas trabajadas a la semana. Por último se solicitó que se clasificara de forma global, del 1 al 5, la cultura de seguridad.

\section{Procedimiento}

Se encuestó a los profesionales sanitarios adscritos a la Unidad de Cardiología, mediante un cuestionario de autocumplimentación. Se incluyó personal fijo e interino, con la intención de lograr que el conocimiento de la realidad de la Unidad fuese lo más amplio posible. El cuestionario no se proporcionó ni a la coordinadora de enfermería ni al jefe de la unidad (como responsables máximos de la implantación de la política de seguridad del paciente). Este sí fue distribuido a los responsables de calidad y seguridad, de formación y docencia, y de calidad. La participación en el estudio fue voluntaria y anónima. El plazo para completarlo fue de una semana. Se distribuyó durante la tercera semana de mayo del 2014 y 2015.

\section{Análisis de los datos}

En un primer momento se efectuó un estudio descriptivo de las dimensiones incluidas en el cuestionario. Además se describieron las variables sociolaborales. Como el cuestionario contiene preguntas formuladas positivamente $y$ otras formuladas negativamente, para el análisis global por dimensiones, se invierte la escala de las preguntas que están formuladas en sentido negativo. Para cada dimensión se obtiene un indicador (porcentaje de respuestas positivas) calculado según la siguiente fórmula:

$\Sigma$ Número de respuestas positivas en los ítems de una dimensión

Número de respuestas totales en los ítems de una dimensión

Para clasificar las fortalezas y oportunidades de mejora se ha seguido el esquema de criterios propuesto por la AHRQ. Se consideró una dimensión como fortaleza cuando el porcentaje de respuestas positivas era mayor o igual al $75 \%$, y como debilidad u oportunidad de mejora cuando el porcentaje de respuestas negativas era mayor o igual al $50 \%$. Para las variables sociolaborales se obtuvieron frecuencias y porcentajes.

\section{Análisis comparativo de los datos}

Hemos comparado el porcentaje de respuestas positivas de cada dimensión en los años 2015 y 2014, así como con los resultados de los hospitales españoles ${ }^{21}$ y de EE. UU. ${ }^{12}$, siguiendo las recomendaciones de la AHRQ. Según estas recomendaciones se considera relevante cualquier diferencia superior al 5\%. Para el análisis de los datos hemos utilizado el programa SPSS stadistics versión 19. 
Tabla 1 La distribución de factores sociolaborales y tasa de respuesta

\begin{tabular}{llllll}
\hline Profesión & $\begin{array}{l}\text { Encuestados } \\
(\%)\end{array}$ & $\begin{array}{l}\text { Tasa de } \\
\text { respuesta } \\
(\%)\end{array}$ & $\begin{array}{l}\text { Más de 6 } \\
\text { años en la } \\
\text { unidad (\%) }\end{array}$ & $\begin{array}{l}\text { Antigüedad más } \\
\text { de 6 años en } \\
\text { profesión (\%) }\end{array}$ & $\begin{array}{l}\text { Trabajo más de } \\
20 \mathrm{~h} \text { semanales } \\
(\%)\end{array}$ \\
\hline Cardiólogo & 18 & 100 & 100 & 100 & 100 \\
Enfermería & 46 & 100 & 100 & 100 & 100 \\
Auxiliares & 26 & 87.5 & 100 & 86 & 100 \\
Apoyo & 10 & 100 & 100 & 67 & 100 \\
\hline
\end{tabular}

Tabla 2 Porcentajes de respuestas positivas, neutras y negativas de cada dimensión. Año 2015

\begin{tabular}{llll}
\hline Dimensión & \%Negativas & \%Neutras & \%Positivas \\
\hline Frecuencia de eventos notificados & 0 & 20 & $80^{\text {a }}$ \\
Percepción de seguridad & 12 & 13 & 74 \\
Expectativas y acciones de la supervisión de la unidad & 0 & 1 & $99^{\text {a }}$ \\
Aprendizaje organizacional/mejora continua & 1 & 3 & $95^{\text {a }}$ \\
Trabajo en equipo en la unidad/servicio & 6 & 12 & $82^{\text {a }}$ \\
Franqueza en la comunicación & 5 & 15 & $80^{\text {a }}$ \\
Feed-back y comunicación sobre errores & 1 & 15 & $84^{\text {a }}$ \\
Respuesta no punitiva a los errores & 7 & 11 & $82^{\text {a }}$ \\
Dotación de personal & 25 & 34 & 41 \\
Apoyo de la gerencia del hospital en la seguridad del paciente & 35 & 34 & 31 \\
Trabajo en equipo entre unidades & 20 & 32 & 19 \\
Problemas en cambios de turno y transiciones entre servicios & 15 & 66 \\
\hline
\end{tabular}

a Fortalezas según la metodología propuesta por la AHRQ.

\section{Resultados}

\section{Tasa de respuesta y factores sociolaborales}

En la tabla 1 se muestra la tasa de respuesta y los factores sociolaborales, en función del estamento, en el año 2015.

\section{Análisis de cultura de seguridad. Fortalezas y oportunidades de mejora}

En cuanto al análisis global de la cultura de seguridad, el conjunto de los encuestados la puntuó con una media de 4.5 en el 2014 y de 4.7 en 2015.

En la tabla 2 se muestra el porcentaje de respuestas positivas, neutras y negativas de cada dimensión analizada en el año 2015.

Se identificaron 7 fortalezas en nuestra unidad: Frecuencia de eventos notificados, Expectativas y acciones de la supervisión de la unidad, Trabajo en equipo en la unidad, Franqueza en la comunicación, Feed-back y comunicación sobre errores y Respuesta no punitiva. No se identificó según la metodología de AHRQ oportunidades de mejora.

\section{Comparación de resultados}

En la tabla 3 se muestra el porcentaje de respuestas positivas de nuestra unidad en 2 años consecutivos así como los resultados del Ministerio de Sanidad del $2009^{21}$ y los de la base de datos de la AHRQ del año $2014^{12}$.
En la figura 1 se puede observar de forma global y gráfica los datos de todas las dimensiones, en la unidad, a nivel nacional y de la base de datos de la AHRQ.

\section{Discusión}

En este trabajo hemos estudiado la cultura de seguridad de una unidad de cardiología, utilizando el cuestionario HSOPSC adaptado y validado para España, analizando un total de 12 dimensiones, y poniendo en evidencia que la unidad presenta una cultura de seguridad considerablemente buena, obteniendo 7 fortalezas, según la metodología propuesta por la AHRQ. Este es el primer estudio sobre cultura de seguridad que se ha realizado en el ámbito de la cardiología.

Nuestra Unidad fue la primera de Cardiología de España en certificar su sistema de gestión de calidad según la norma ISO 9001. Posteriormente también se certificó la gestión de riesgos para la seguridad del paciente según la norma UNE 179003 , siendo el tercero del país y el primero en el ámbito de la cardiología 28 .

Los resultados mostraron que la cultura de seguridad en nuestra unidad fue muy alta, tanto en la evaluación del año 2014 como en el año 2015. En el año 2015, se identificaron 7 fortalezas dentro del análisis (Frecuencia de eventos notificados, Expectativas y acciones de la dirección/supervisión de la unidad/servicio que favorecen la seguridad, Aprendizaje organizacional/mejora continua, Trabajo en equipo en la unidad/servicio, Franqueza en la comunicación, Feedback y comunicación sobre errores y Respuesta no punitiva a los errores). Adicionalmente, la dimensión Percepción 
Tabla 3 Porcentaje de respuestas positivas en cada dimensión en los diferentes estamentos comparados

\begin{tabular}{lllll}
\hline & Unidad 2015 & Unidad 2014 & Nacional 2009 & EE. UU. 2014 \\
\hline Frecuencia de eventos notificados & 80 & 81 & 46 & $66(90)$ \\
Percepción de seguridad & 74 & 73 & 47 & $66(75)$ \\
Expectativas y acciones de la supervisión & 99 & 91 & 61 & $76(100)$ \\
Aprendizaje organizacional/mejora continua & 95 & 96 & 54 & 71 \\
Trabajo en equipo en la unidad/servicio & 82 & 91 & 48 & $81(100)$ \\
Franqueza en la comunicación & 80 & 92 & 43 & $62(90)$ \\
Feed-back y comunicación sobre errores & 84 & 88 & 53 & $67(90)$ \\
Respuesta no punitiva a los errores & 82 & 42 & 26 & $44(100)$ \\
Dotación de personal & 41 & 39 & 24 & $55(10)$ \\
Apoyo de la gerencia del hospital en la seguridad & 31 & 52 & 42 & $72(10)$ \\
Trabajo en equipo entre unidades & 48 & 70 & 53 & $61(10)$ \\
Problemas en cambios de turno y transiciones & 66 & $47(90)$ & \\
\hline
\end{tabular}

Entre paréntesis, percentil que ocupa nuestra unidad dentro de la base de datos de la AHRQ.

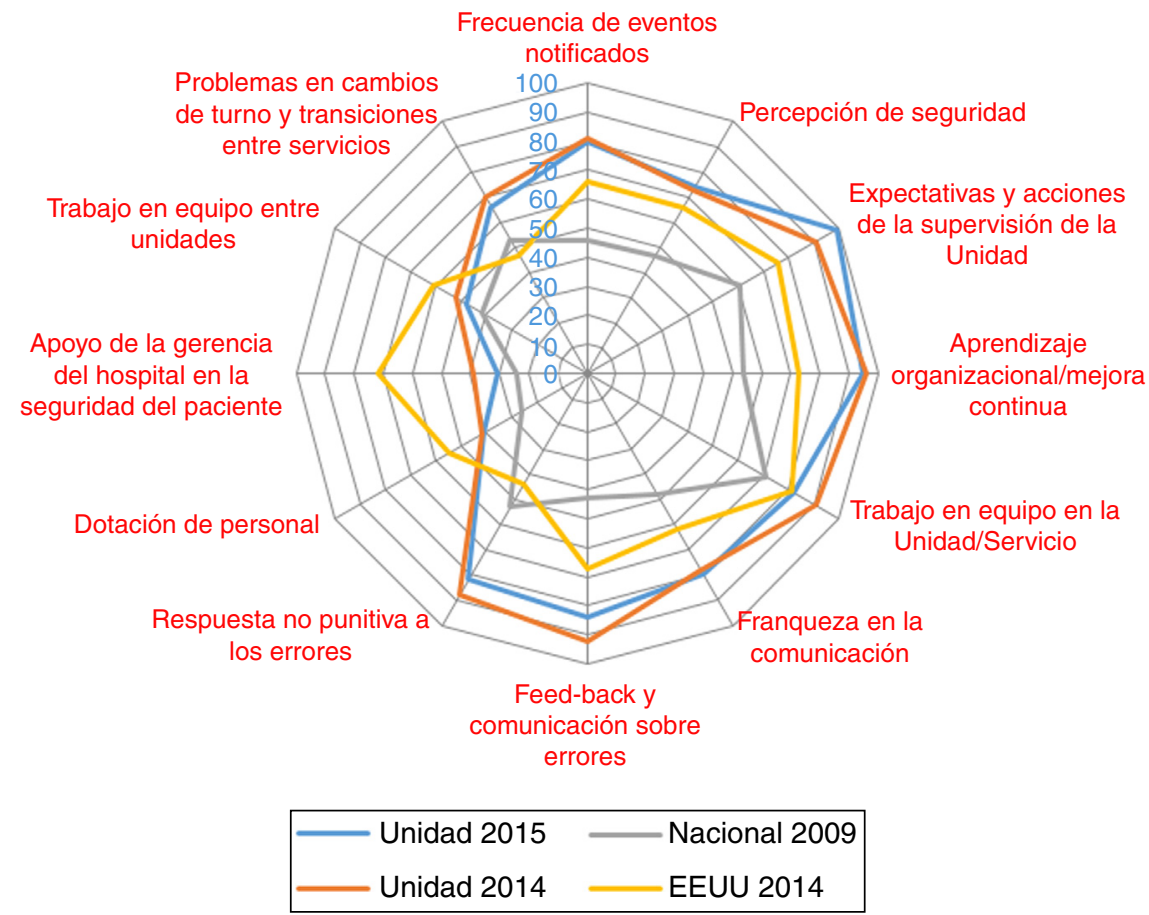

Figura 1 Porcentaje de respuestas positivas de cada dimensión, en los diferentes ámbitos analizados.

de seguridad se encontró muy cercana a ser considerada una fortaleza con un $74 \%$ de respuestas positivas. No se han identificado oportunidades de mejora, sin embargo las dimensiones sobre Dotación de personal, Apoyo de la gerencia y Trabajo en equipo entre unidades fueron las peor valoradas. La valoración global de la cultura de seguridad fue muy positiva, obteniendo una puntuación media de 4.7 sobre 5.

Si analizamos la evolución entre los años 2014 y 2015, dentro de la unidad, podemos decir que los resultados fueron muy similares. La valoración global de la cultura de seguridad subió 2 décimas, de 4.5 a 4.7. Tras aplicar la metodología de la AHRQ se mejoró en la dimensión de Expectativas y acciones de la supervisión de la unidad (99 vs. 91\%) y se empeoró relativamente en las dimensiones de Trabajo en equipo (88 vs. 91\%), Feed-back y comunicación sobre errores ( 84 vs. $92 \%$ ), Respuestas no punitivas a los errores ( 82 vs. $88 \%$ ) y Apoyo de la gerencia (31 vs. $39 \%$ ). Si bien las variaciones en estas dimensiones son superiores al $5 \%$, no existieron desviaciones que en nuestra opinión se pudieran considerar relevantes, pese a que en abril del año 2014 nos certificamos según la norma UNE 179003:2013 sobre gestión de riesgos para la seguridad del paciente. La aplicación de esta norma supuso una mayor concienciación del personal sobre temas de seguridad del paciente, y por lo tanto un mayor grado de autoevaluación y autocrítica. Ello puede suponer un empeoramiento en la valoración de la cultura de seguridad que en nuestro caso no se produjo, cosa que es valorada de forma muy positiva. Por otra parte, que los datos fueran muy similares en 2 años consecutivos habla a favor de la 
buena reproducibilidad del cuestionario que se ha utilizado para el estudio.

Al realizar la comparación a nivel nacional, las diferencias fueron muy marcadas. Dicha comparación se realizó con hospitales de tamaño mediano como el nuestro, si bien la encuesta nacional de 2009 se llevó a cabo en varias especialidades dentro de los diferentes hospitales ${ }^{21}$. Los resultados mostraron que las valoraciones en todas las dimensiones analizadas son claramente superiores en nuestra unidad. Cabe destacar que las dimensiones peor valoradas son la dotación de personal (el $26 \%$ de respuestas positivas) y el apoyo de la gerencia (el $24 \%$ de respuestas positivas). Estas 2 dimensiones fueron las peores valoradas también en nuestro análisis.

Como ya se ha comentado, en EE. UU. la AHRQ analiza la cultura de seguridad de sus hospitales cada 2 años ${ }^{12}$. En dicho país la cultura de seguridad está mucho más arraigada que en el nuestro. Sin embargo en 8 dimensiones nuestros resultados fueron superiores (Frecuencia de eventos notificados, Percepción de seguridad, Expectativas y acciones de la supervisión de la unidad, Aprendizaje organizacional/mejora continua, Franqueza en la comunicación, Feed-back y comunicación sobre errores, Respuesta no punitiva a los errores y Problemas en cambios de turno y transiciones entre servicios). Existieron 3 dimensiones peor valoradas en nuestro análisis (Dotación de personal, Apoyo de la gerencia y Trabajo en equipo entre unidades). Esto se podría deber a las diferencias organizativas del sistema de salud americano basado en compañías aseguradoras.

Si bien de forma estricta no se identificaron dimensiones como oportunidades de mejora, pensamos que hay que mejorar en aquellas peor evaluadas, y que coinciden con las peor valoradas a nivel nacional y en otros estudios similares. Es por ello de vital importancia prestar atención a la racionalidad de la dotación y organización del personal y los ritmos de trabajo a que se puedan ver sometidos. Sobre este aspecto sería importante la sensibilización de las direcciones de hospital en vistas a realizar una adecuación de las plantillas a la presión laboral que soportan. Es importante mostrar una actitud más claramente proactiva hacia la seguridad del paciente por parte de las gerencias. La seguridad del paciente debería de ser un pilar estratégico de la organización y no solo iniciativas individuales de algún servicio; de forma que se debería fomentar la responsabilidad compartida y la coordinación entre unidades y servicios. En los resultados del estudio se percibió la necesidad de mejorar la coordinación entre unidades-servicios y debería por tanto desarrollarse una estrategia tendente a hacer extensiva la sensación de trabajo en equipo que existe dentro de la unidad entre todas las unidades.

La principal limitación de nuestro estudio fue que se analiza únicamente una unidad de un hospital de mediano tamaño. Es por ello que el número de encuestas analizadas es pequeño respecto a otros estudios. Sin embargo hay que tener en cuenta que en nuestro caso se analizó a toda la población, obteniendo una tasa de respuesta del $97 \%$. No existió pues sesgo de no respuesta, a diferencia de otros estudios.

Aunque existen trabajos que demuestran que aquellas unidades con mayor cultura de seguridad obtienen mejores resultados en sus pacientes ${ }^{13,14}$, en el ámbito de la cardiología no hemos encontrado ningún estudio que analice este aspecto. Sin embargo parece lógico pensar que las instituciones que establecen medidas tendentes a garantizar al máximo la seguridad de los pacientes podrían obtener resultados más favorables. Por otro lado, la implantación de sistemas de gestión de la calidad ha demostrado beneficio en diferentes aspectos de atención al paciente. Existe evidencia de que el seguimiento y la monitorización de los procesos que se realizan en una unidad suponen una mejora continua en los servicios prestados a nuestros pacientes ${ }^{25-27}$.

\section{Conclusiones}

Con todo ello concluimos que la cultura de seguridad en una unidad de cardiología con un sistema integrado de gestión de calidad y riesgos y seguridad del paciente es elevada, superior a la nacional en todas sus dimensiones y en la mayoría de ellas respecto al registro de EE. UU. Esto indica la conveniencia de aumentar implantación de este tipo de sistemas de gestión y de promover más estudios orientados a la evaluación de la cultura de seguridad.

\section{Responsabilidades éticas}

Protección de personas y animales. Los autores declaran que para esta investigación no se han realizado experimentos en seres humanos ni en animales.

Confidencialidad de los datos. Los autores declaran que han seguido los protocolos de su centro de trabajo sobre la publicación de datos de pacientes.

Derecho a la privacidad y consentimiento informado. Los autores declaran que en este artículo no aparecen datos de pacientes.

\section{Financiación}

Ninguna.

\section{Conflicto de intereses}

Los autores declaran no tener ningún conflicto de intereses

\section{Bibliografía}

1. Nieva VF, Sorra J. Safety culture assessment: A tool for improving patient safety in healthcare organizations. Qual Saf Health Care. 2003;12 Suppl 2:ii17-23.

2. The National Quality Forum: Safe Practices for Better Healthcare. A Consensus report. Washington: NQF; 2003.

3. The National Quality Forum. Safe Practices for Better Health Care, 2006 Update. Washington: NQF; 2007.

4. Consejo de Europa. La seguridad del paciente: un reto europeo. Varsovia, 1315. Abril 2005.

5. Agencia de Calidad del Sistema Nacional de Salud. Estrategia para la seguridad del paciente: recomendaciones del taller de expertos celebrado el 8 y 9 de febrero de 2005. Madrid: Ministerio de Sanidad y Consumo; 2005.

6. Ministerio de Sanidad, Política Social e Igualdad. Plan de calidad para el Sistema Nacional de Salud. Madrid: Ministerio de Sanidad, Política Social e Igualdad; 2010. 
7. Colla JB, Bracken AC, Kinney LM, et al. Measuring patient safety climate: A review of surveys. Qual Saf Health Care. 2005; $14: 364-6$.

8. Flin R, Burns C, Mearns $\mathrm{K}$, et al. Measuring safety climate in health care. Qual Saf Health Care. 2006;15:109-15.

9. Sexton JB, Helmreich RL, Neilands TB, et al. The Safety Attitudes Questionnaire: Psychometric properties, benchmarking data, and emerging research. BMC Health Serv Res. 2006;6:44.

10. Hospital Survey on Patient Safety Culture. February 2015. Rockville, MD: Agency for Healthcare Research and Quality [consultado 23 Mar 2016]. Disponible en: http: / / www.ahrq.gov/professionals/quality-patient-safety/ patientsafetyculture/hospital/index.html

11. Cuestionario sobre seguridad de los pacientes: versión española del Hospital Survey on Patient Safety. Madrid: Ministerio de Sanidad y Consumo; 2005.

12. Hospital Survey on Patient Safety Culture: 2014 user comparative database report. Rockville, MD: Agency for Healthcare Research and Quality [consultado 23 Mar 2016]. Disponible en: http://www.ahrq.gov/professionals/quality-patient-safety/ patientsafetyculture/hospital/index.html

13. Marsteller JA, Wen M, Hsu Y-J, et al. Safety culture in cardiac surgical teams: Data from five programs and national surgical comparison. Ann Thorac Surg. 2015;100:2182-9.

14. Birkmeyer NJO, Finks JF, Greenberg CK, et al. Safety culture and complications after bariatric surgery. Ann Surg. 2013;257:260-5.

15. Singer S, Meterko M, Baker L, et al. Workforce perceptions of hospital safety culture: Development and validation of the patient safety climate in healthcare organizations survey. Health Serv Res. 2007;42:1999-2021.

16. Singer SJ, Gaba DM, Falwell A, et al. Patient safety climate in 92 US hospitals: Differences by work area and discipline. Med Care. 2009;47:23-31.

17. Hellings J, Schrooten W, Klazinga N, et al. Challenging patient safety culture: Survey results. Int J Health Care Qual Assur. 2007;20:620-32.
18. Olsen E. Workers' perceptions of safety culture at a hospital. Tidsskr Prakt Med ny række. 2007;127:2656-60.

19. Bodur S, Filiz E. Validity and reliability of Turkish version of Hospital Survey on Patient Safety Culture and perception of patient safety in public hospitals in Turkey. BMC Health Serv Res. 2010;10:28.

20. Saturno PJ, da Silva Gama ZA, de Oliveira-Sousa SL, et al. Análisis de la cultura sobre seguridad del paciente en los hospitales del Sistema Nacional de Salud español. Med Clin (Barc). 2008;131:18-25.

21. Análisis de la cultura sobre seguridad del paciente en el ámbito hospitalario del Sistema Nacional de Salud Español. Madrid: Ministerio de Sanidad y Protección social; 2009.

22. Pozo F, Padilla V. Evaluación de la cultura de seguridad del paciente en el ámbito de un área sanitaria. Rev Calid Asist. 2013;28:329-36.

23. Astier MP, Torijano ML, Olivera G, et al. Are Spanish primary care professionals aware of patient safety? Eur J Public Health. 2015;25:781-7.

24. Ulecia MÁ, Gómez JJ. La calidad en cardiología. Desde la teoría a la aplicación práctica. Rev Española Cardiol Supl. 2007; 7:47C-54C.

25. Cortés MA, Irrazábal E, García-Jerez A, et al. Impact of implementing ISO 9001:2008 standard on the Spanish Renal Research Network biobank sample transfer process. Nefrologia. 2014;34:1-9.

26. Shaw CD, Groene O, Botje D, et al. The effect of certification and accreditation on quality management in 4 clinical services in 73 European hospitals. Int J Qual Health Care. 2014;26 Suppl $1: 100-7$.

27. Stoimenova A, Stoilova A, Petrova G. ISO 9001 certification for hospitals in Bulgaria: Does it help service? Biotechnol Biotechnol Equip. 2014;28:372-8.

28. Merino P, Bustamante E, Campillo C, et al. Certificación en seguridad del paciente en un Servicio de Medicina Intensiva: nuestra experiencia con la norma UNE 179003:2013. Med Intensiva. 2014;38:297-304. 